\title{
miR-494 promotes progression of retinoblastoma via PTEN through PI3K/AKT signaling pathway
}

\author{
FEN XU ${ }^{1}$, GUIQIN LIU ${ }^{2}$, LIJUAN WANG $^{3}$, XIYAN WANG $^{4}$, XIAO JIN $^{5}$ and WEN BO ${ }^{6}$ \\ Departments of ${ }^{1}$ Clinical Laboratory and ${ }^{2}$ Ophthalmology, Jinan Zhangqiu District Hospital of TCM; \\ Departments of ${ }^{3}$ Paediatrics, ${ }^{4}$ Anesthesiology and ${ }^{5}$ Rehabilitation Medicine, The People's Hospital of Zhangqiu Area, \\ Jinan, Shandong 250200; ${ }^{6}$ Department of Ophthalmology, Maternity and Child Health Care of Zaozhuang, \\ Zaozhuang Ophthalmological Hospital, Zaozhuang, Shandong 277100, P.R. China
}

Received August 9, 2019; Accepted March 10, 2020

DOI: $10.3892 / \mathrm{ol} .2020 .11749$

\begin{abstract}
Increasing evidence has indicated that the dysregulation of microRNA (miRNA) occur in the pathogenesis of retinoblastoma $(\mathrm{RB})$. Aim of the present study was to investigate the possible role of miR-494 (miR-494-3p) in RB. It was demonstrated that miR-494 expression was increased in RB tissue samples and cell lines. Also, it was prominently associated with clinicopathological features. Functional assays showed that RB cell proliferation, invasion and migration can be promoted by miR-494 overexpression. Besides, phosphatase and tensin homolog (PTEN) was verified as a possible target of miR-494 by a luciferase assay, western blot and qRT-PCR assay in RB. miR-494 and PTEN expression was negatively related in a correlation analysis on tumor tissues of 66 patients. In addition, PTEN was proved to reverse miR-494 effect on RB cell progression. Moreover, PI3K/AKT signaling pathway was validated to take part in $\mathrm{RB}$ progression. Taken together, the current study proposes that miR-494 might function as a tumor promoter and regulates RB progression through targeting PTEN.
\end{abstract}

\section{Introduction}

Retinoblastoma ( $\mathrm{RB}$ ) is the most prevalent type of primary inherited intraocular malignant tumor in infancy and children, affecting their life, vision, facial appearance and psychological development $(1,2)$. The disease is prone to intracranial and distant metastasis, often endangering the life of the child, particularly in children aged $<5$ years $(3,4)$. The predominant treatment for patients includes chemotherapy, enucleation, laser therapy or cryotherapy, the most common

Correspondence to: Dr Wen Bo, Department of Ophthalmology, Maternity and Child Health Care of Zaozhuang, Zaozhuang Ophthalmological Hospital, 25 Wenhua East Road, Zaozhuang, Shandong 277100, P.R. China

E-mail: qukangjinggu6673@163.com

Key words: retinoblastoma, miR-494, PTEN, PI3K/AKT pathway treatment protocol includes chemotherapy combined with immunotherapy, which is cytotoxic to RB cells (1). Therefore, early detection, early diagnosis and early treatment are the key to improving the cure rate and reducing the mortality rate.

MiRNAs, a family of small and noncoding RNA molecules consisting of 21-25 nucleotides, regulate the expression of their target genes through suppressing the translation or promoting the RNA degradation $(5,6)$. They are identified as tumor suppressors or oncogenes in many types of tumors and thus regulate tumor progression and metastasis. Mounting evidence has indicated that miRNAs were identified in $\mathrm{RB}$ and regulated cell migration, invasion, proliferation and apoptosis (7-11). Sun et al (12) showed that suppression of miR-492 inhibited RB cell viability and invasion by targeting LATS2. miR-183 may be involved in RB progression by targeting LRP6 (13). Also, miR-34a exhibited inhibitory effect on RB cell growth and promoting effect on cell apoptosis (14). Moreover, miR-494 (miR-494-3p) was identified to be highly expressed in RB cells and associated with its tumorigenesis (15). However, the mechanism regulating RB progression by miR-494 expression is yet to be fully elucidated.

Phosphatase and tensin homolog (PTEN) has been reported to regulate the PI3K/AKT signaling pathway negatively (16). Studies have confirmed that PTEN was disordered in multiple cancers, and was considered to be one of the potential factors in tumorigenesis $(17,18)$. Previous studies have summarized the role of miRNAs in regulating PTEN in cancers and have determined that the miRNA/PTEN pathway is involved in tumor cell growth, invasion and migration (19-21). For instance, miR-296/PTEN axis facilitated gastric cancer tumorigenesis in vitro and in vivo (22). miR-548 promoted NSCLC cell invasion by regulating PTEN (23). Moreover, miR-182 promoted breast cancer cell viability and invasion via targeting PTEN (24). A previous study has shown that miR-494-3p can promote the progression of endometrial cancer by regulating the PTEN/PI3K/AKT pathway (25). However, whether PTEN acted as the target of miR-494 in regulating RB progression is unclear.

Herei, it is suggested that miR-494 expression was upregulated in RB tissues, and cells and miR-494 enhanced RB cell viability, migration and invasion. PTEN was verified as the 
target of miR-494 in RB cells. I was shown that miR-494 facilitated RB cell progression by targeting PTEN through PI3K/AKT signaling pathway.

\section{Patients and methods}

Tissue samples. Ten normal retina tissues and RB tissues were provided by Jinan Zhangqiu District Hospital of TCM (Jinan, China). None of the patients in this study received preoperative radiotherapy or chemotherapy, and all patients were diagnosed by three pathologists. The patients consisted of 6 males and 4 females aged 1.2 to 9.9 years (mean, 5.4). According to ICRB, the RB patients were divided into phase I ( 3 cases), phase II ( 5 cases) and phase III ( 2 cases). There was no significant difference in age, sex and clinical stages of the 10 patients. The adjacent healthy tissues were set as the control. The fresh samples were stored in $-80^{\circ} \mathrm{C}$ refrigerator for further analysis. Parents of the patients provided a written informed consent prior to surgical excision. The Ethics Committee of Jinan Zhangqiu District Hospital of TCM approved this study.

Cell culture. Two RB cell lines (Y79, SO-RB50) and normal retina epithelium cell line (APRE-19) were obtained from ATCC. All the cells were cultured in RPMI-1640 medium with $10 \%$ FBS. Cells were then left to grow in a humidified incubator containing $5 \% \mathrm{CO}_{2}$ at $37^{\circ} \mathrm{C}$.

$q R T-P C R$. Total RNA was extracted with the help of TRIzol reagent (Invitrogen; Thermo Fisher Scientific, Inc.). The concentration and purity of RNA were tested via NanoDrop 2000 instrument (Thermo Fisher Scientific, Inc.). To perform the quantitative detection of miR-18a or mRNA expression, cDNAs were synthesized through the PrimeScript RT reagent (Takara Biotechnology Co.). GAPDH was used as the control for the normalization of expression levels of genes. The snRNA U6 was used as the control of miRNA. The primers were as follows: miR-494-F, 5'-GATACTCGAAGGAGAGGTTGTC-3' and miR-494-R, 5'-GAGGTTTCCCGTGTATGTTTCAT-3'; PTEN-F, 5'-CGGCAGCATCAAATGTTTCAG-3' and PTEN-R, 5'-AACTGGCAGGTAGAAGGCAACTC-3'; GAPDH-F, 5'-CTCTGCTCCTCCTGTTCGAC-3' and GAPDH-R, 5'-CGA CCAAATCCGTTGACTCC-3'; U6-F, 5'-ATTGGAACGATA CAGAGAAGATT-3' and U6-R, 5'-GGAACGCTTCACGAAT TTG-3'. The data were expressed as $2^{-\Delta \Delta C t}$, indicating the target mRNA relative level.

Western blot analysis. Total protein was extracted with RIPA lysis buffer. The protein concentration was determined using the BCA method (Beyotime Institute of Biotechnology). The assay was performed as follow: $50 \mu \mathrm{g}$ of total proteins were loaded onto the gel and electrophoresed. After transferring to the $\mathrm{NC}$ membranes, the membranes were blocked with $5-10 \%$ skim milk. Then, the membranes were incubated with the primary antibodies at $4^{\circ} \mathrm{C}$ overnight. Subsequently, the second antibodies were added for incubating at room temperature for $2 \mathrm{~h}$. Finally, ECL agents were applied for observing the protein bands. ImageJ software (version 1.48; National Institutes of Health) was used for densitometry. GAPDH was employed as an internal control.
MTT assay. RB cells $\left(5 \times 10^{3}\right)$ were placed onto 96 -well plates. When the cells were cultured for $0,1,2,3$ and 4 days, MTT solution $(20 \mu \mathrm{l})$ was added and cultured for $4 \mathrm{~h}$ at $37^{\circ} \mathrm{C}$. A Bio-Rad microplate reader (Bio-Rad Laboratories, Inc.) was used to read the absorbance at $490 \mathrm{~nm}$.

Overexpression of $\mathrm{miR}-494$ or silence $\mathrm{miR}-494$ and overexpression of PTEN. miR-494 mimic or inhibitor was purchased from RiboBio Co., Ltd. SiRNA PTEN was obtained from Genechem Co., Ltd. The transfection was performed with the help of Lipofectamine 2000 for $48 \mathrm{~h}$.

Transwell assay. The Transwell assay was applied to perform cell migration and invasion using Transwell inserts $(8 \mu \mathrm{m}$ pores; BD Biosciences). The differences between cell migration and invasion were whether the top chamber was pre-coated with or without Matrigel. Firstly, the cells were placed into the top chamber to incubate for $24 \mathrm{~h}$. The lower chamber was added with complete medium with $10 \%$ FBS. The cells in the lower chamber that migrated or invaded from the top chamber were then fixed with $4 \%$ paraformaldehyde and stained with $0.5 \%$ crystal violet. Finally, the number of migrated or invaded cell was counted using a microscope (magnification, x200).

Luciferase assay. The wild-type (wt) and mutant (mut) 3'UTR of PTEN was first inserted into the pGL3 reporter vector (Promega Corporation). Then, the PTEN 3'UTR-pGL3 reporter vector and $\mathrm{miR}-494 \mathrm{mimic}$ were co-transfected into Y79 cells and plated in 24-well plates for $48 \mathrm{~h}$, the cells were harvested and lysed, and the luciferase activity was detected by a Dual-Luciferase Reporter System (Promega) according to the protocol of the manufacturer. Relative firefly luciferase activity was normalized to Renilla luciferase activity.

Statistical analysis. Results are represented as the mean \pm SD of at least triplicates. SPSS 22.0 Software (IBM Corp.) was applied for performing statistical analyses. Student's t-test was carried out for determining the statistical significance of differences in two groups and one-way analysis of variance with Tukey's post hoc test was applied in more than two groups. $\mathrm{P}<0.05$ indicates statistical significance.

\section{Results}

Increase of miR-494 in RB tissues. The average expression level of miR-494 in RB tissues was analyzed by qRT-PCR and the findings indicated that it was obviously increased in RB tissue samples compared with normal tissues (Fig. 1A). Then miR-494 expression level was evaluated in 5 clinical RB specimens versus 5 normal tissues. Results showed that its expression was obviously increased in RB tissues (Fig. 1B). Moreover, the correlation between miR-494 expression and the clinicopathological features of $66 \mathrm{RB}$ patients was analysed. The median value of miR-494 expression was used as the cut-off point to divide miR-494 expression into high and low expression of miR-494. Results demonstrated that the high miR-494 expression was associated with $\mathrm{N}$ classification and differentiation in patients with RB (Table I). Collectively, these results indicated that miR-494 might play important roles in RB progression. 

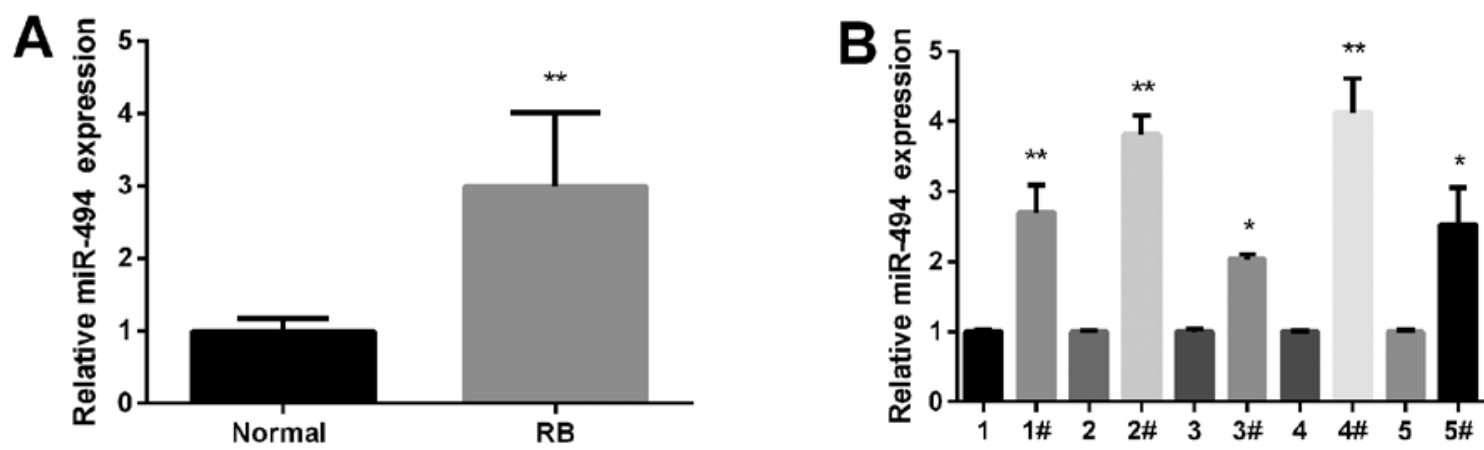

Figure 1. Upregulation of miR-494 in RB tissue samples. (A) Average expression level of miR-494 tested in RB tissue samples by qRT-PCR (n=10). (B) Expression level of miR-494 in $5 \mathrm{RB}$ tissue specimens by qRT-PCR. ${ }^{*} \mathrm{P}<0.05,{ }^{* * *} \mathrm{P}<0.01$. RB, retinoblastoma.

miR-494 enhances $R B$ cell viability, invasion and migration. Two RB cell lines (Y79, SO-RB50) were applied to investigate miR-494 function in RB progression. Due to the higher expression of miR-494 in Y79 cells, miR-494 expression was increased in $\mathrm{Y} 79$ cells, and miR-494 expression in SO-RB50 cells was suppressed (Fig. 2A). qRT-PCR was applied for confirming efficiency of miR-494 transfection in the two cell lines respectively (Fig. 2B). Then, MTT assay was performed to detect cell viability affected by altered miR-494. It was found that increasing miR-494 enhanced Y79 cell paoliferation, while decreasing miR-494 inhibited SO-RB50 cells viability (Fig. 2C). Furthermore, Transwell assay was used to investigate miR-494 effect on cell invasion and migration. Data showed dramatically increased mobility and invasiveness of Y79 cells by miR-494 mimic, whereas it was markedly reduced by miR-494 inhibitor in SO-RB50 cells (Fig. 2D and E).

PTEN is the miR-494 target gene in RB cells. We used the algorithm provided by TargetScan Human to predict the candidate targets of miR-494 and to determine the mechanisms responsible for the effects of miR-494 in RB cells. PTEN was temporarily selected for further validation among the predicted targets, because it has a potential role in the regulation of RB malignancies (Fig. 3A). To further verify whether PTEN was the direct target of miR-494, Luciferase-based assay was carried out to perform this experiment. The findings showed that miR-494 mimic dramatically inhibited PTEN 3'UTR luciferase activity in Y79 cells (Fig. 3B). Consistently, miR-494 inhibitor dramatically enhanced PTEN 3'UTR luciferase activity in SO-RB50 cells (Fig. 3C). Apart from this, western blot analysis and qRT-PCR were performed to test PTEN expression. Results indicated that PTEN protein (Fig. 3D) and mRNA (Fig. 3E) levels were remarkably inhibited by miR-494 overexpression in Y79 cells, and increased by miR-494 suppression in SO-RB50 cells. Fig. 3F shows the negative relationship between miR-494 and PTEN expression by Spearman's Rank correlation.

miR-494 promotes RB cell progression via PTEN. To further detect whether PTEN regulates miR-494 effect on RB cell viability, invasion and metastasis, PTEN level was restored in Y79 cells inhibited by miR-494 overexpression (Fig. 4A). qRT-PCR was applied for confirming efficiency of PTEN transfection in Y79 cells. Then, MTT assay was performed to test cell viability.
Table I. Association study, and correlation between miR-494 expression and clinicopathological characteristics.

\begin{tabular}{|c|c|c|c|c|}
\hline \multirow[b]{2}{*}{ Characteristics } & \multirow[b]{2}{*}{$\mathrm{N}=66$} & \multicolumn{2}{|c|}{ miR-494 } & \multirow[b]{2}{*}{ P-value } \\
\hline & & $\begin{array}{c}\text { Low } \\
\text { expression }\end{array}$ & $\begin{array}{c}\text { High } \\
\text { expression }\end{array}$ & \\
\hline Age (years) & & & & 0.569 \\
\hline$\leq 5$ & 52 & 18 & 34 & \\
\hline$>5$ & 14 & 6 & 8 & \\
\hline Sex & & & & 0.930 \\
\hline Male & 39 & 14 & 25 & \\
\hline Female & 27 & 10 & 17 & \\
\hline Clinical stage & & & & 0.478 \\
\hline I-II & 20 & 6 & 14 & \\
\hline III-IV & 46 & 18 & 28 & \\
\hline $\mathrm{N}$ classification & & & & $0.019^{\mathrm{a}}$ \\
\hline No & 29 & 6 & 23 & \\
\hline $\mathrm{N} 1+2$ & 37 & 18 & 19 & \\
\hline Differentiation & & & & $0.047^{\mathrm{a}}$ \\
\hline Well and moderate & 24 & 5 & 19 & \\
\hline Poorly & 42 & 19 & 23 & \\
\hline Largest tumor (mm) & & & & 0.070 \\
\hline$\leq 15$ & 40 & 18 & 22 & \\
\hline$>15$ & 26 & 6 & 20 & \\
\hline
\end{tabular}

Statistical analyses were performed by the $\chi^{2}$ test. ${ }^{\mathrm{a}} \mathrm{P}<0.05$ was considered significant.

As expected, miR-494 mimic increased the cell proliferation, but its effect was overturned by overexpression of PTEN (Fig. 4B). Similarly, increasing the expression of PTEN also reversed the increased migration (Fig. 4C) and invasion (Fig. 4D) regulated by miR-494 mimic. All the results demonstrated that miR-494 promoted RB cell proliferation, invasion and migration via inhibiting PTEN expression.

miR-494 regulates PI3K/AKT signaling pathway via PTEN. PTEN has been reported to negatively regulate PI3K/AKT signaling pathway. 
A

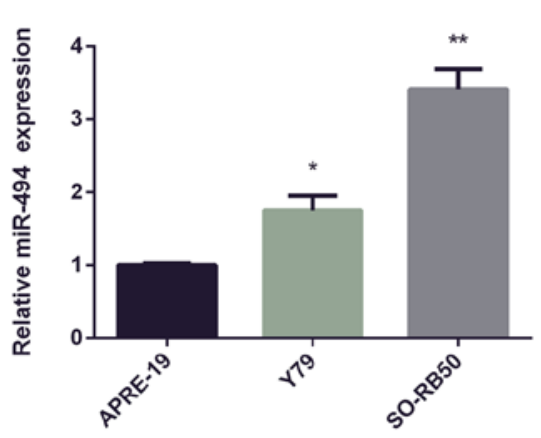

C

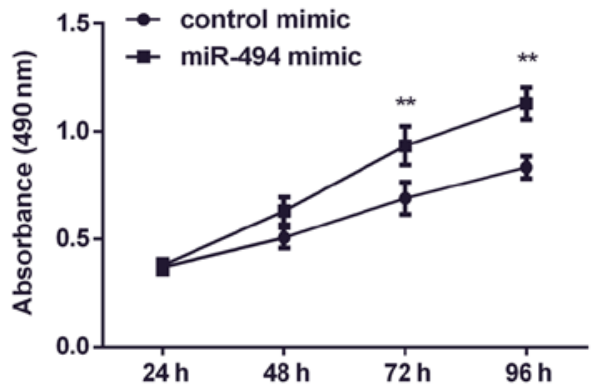

B

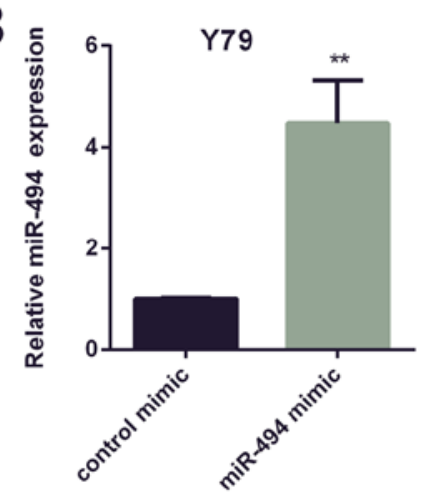

SO-RB50

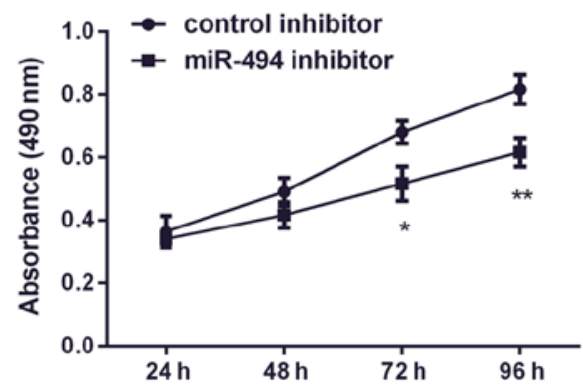

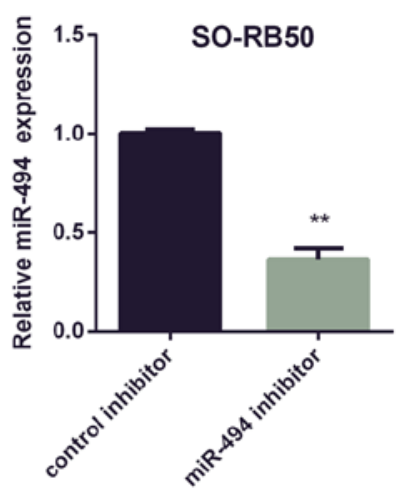

D control mimic miR-494 mimic
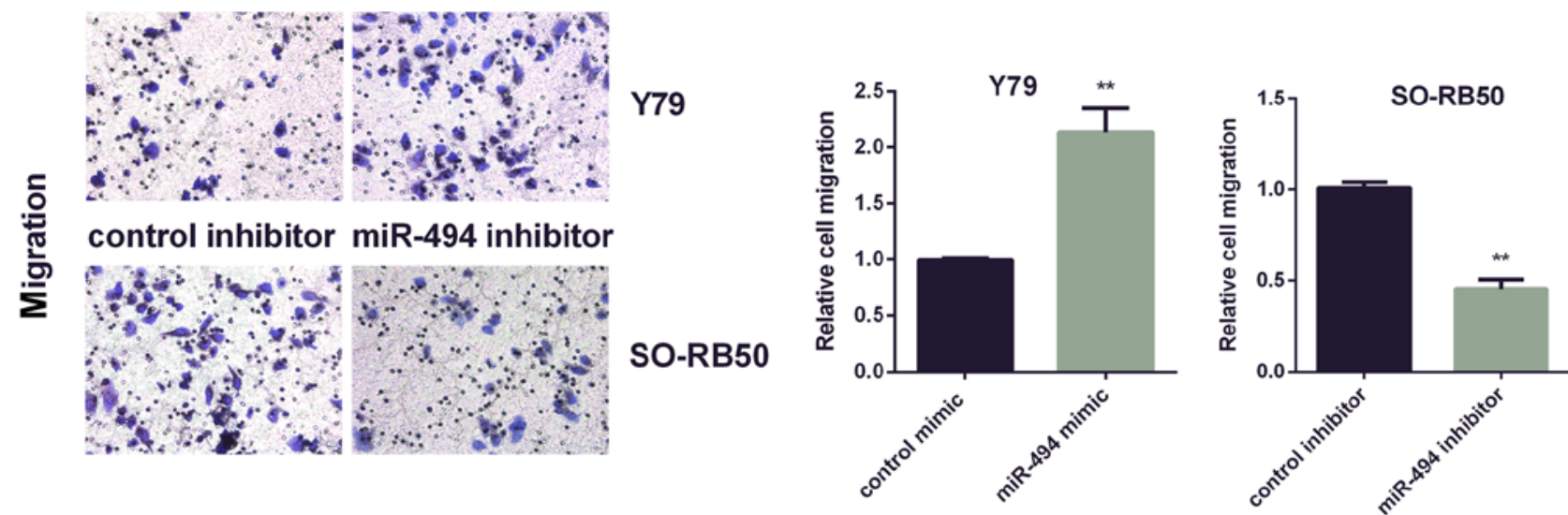

$\mathbf{E}$ control mimic miR-494 mimic
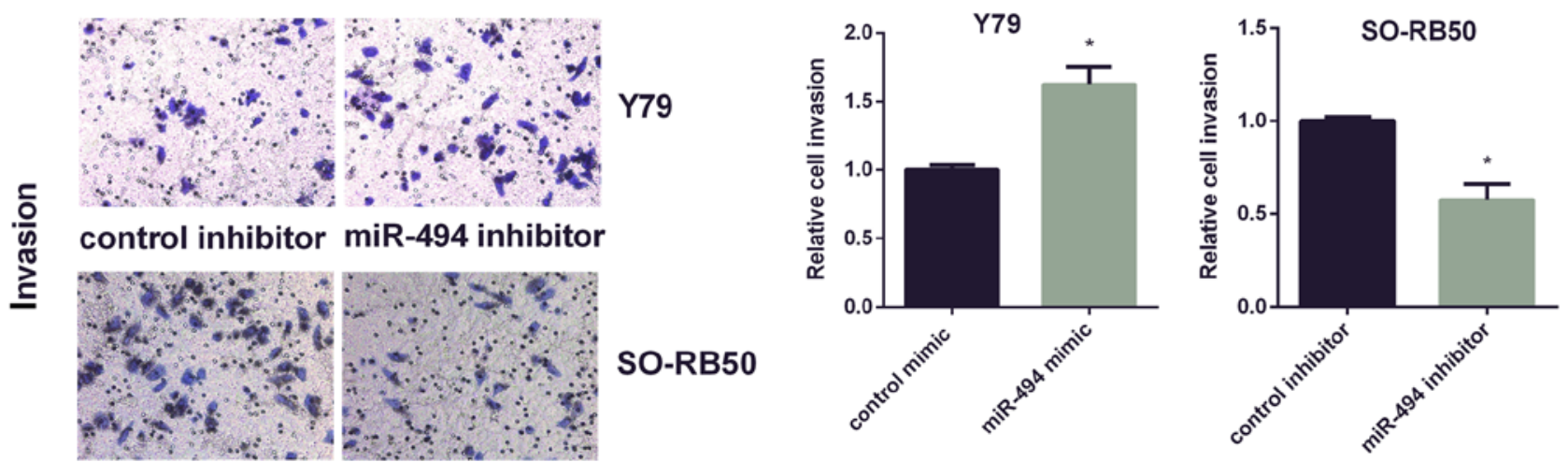

Figure 2. Promotion effect of miR-494 on RB cell progression. (A) qRT-PCR analysis of miR-494 expression level in Y79 and SO-RB50 cells. (B) The expression level of miR-494 tested in Y79 cells after increasing miR-494 and in SO-RB50 cells after decreasing miR-494 by qRT-PCR. (C) Y79 cell viability was measured after increasing miR-494 or SO-RB50 cell viability after inhibiting miR-494 expression by MTT assay. (D) The ability of Y79 cell migration was detected after increasing miR-494 or SO-RB50 cell migration was detected after inhibiting miR-494 expression by Transwell assay. (E) The ability of Y79 cell invasion was measured after increasing miR-494 or SO-RB50 cell invasion and was detected after decreasing miR-494 expression by Transwell assay. ${ }^{*} \mathrm{P}<0.05,{ }^{* * *} \mathrm{P}<0.01$. RB, retinoblastoma. 
A

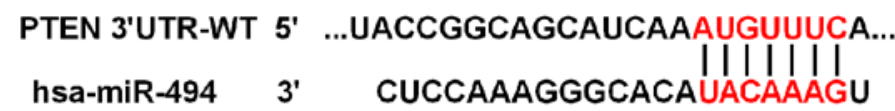

PTEN 3'UTR-MUT 5' ...UACCGGCAGCAUCAAAUGUUUCA...

B

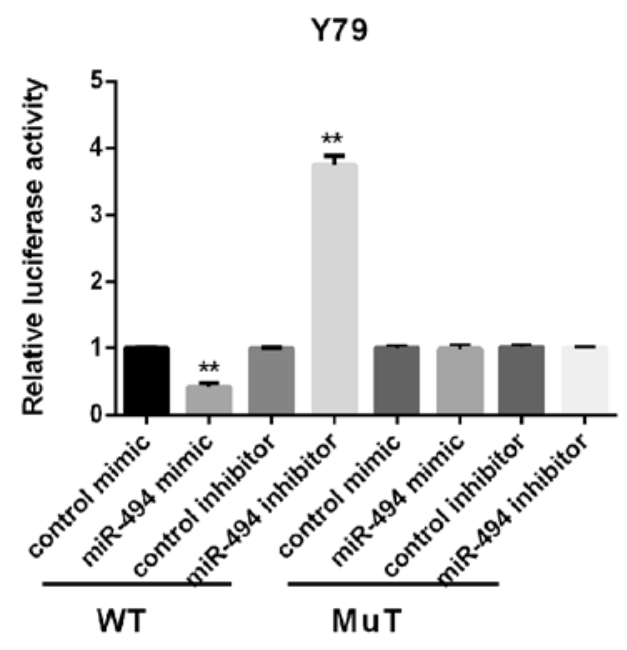

D

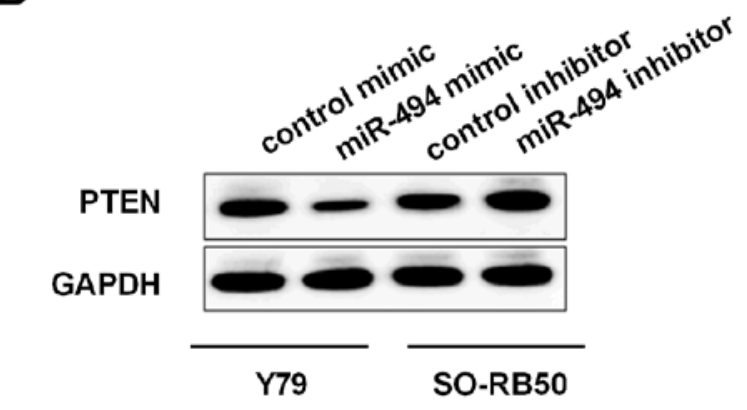

$\mathbf{F}$

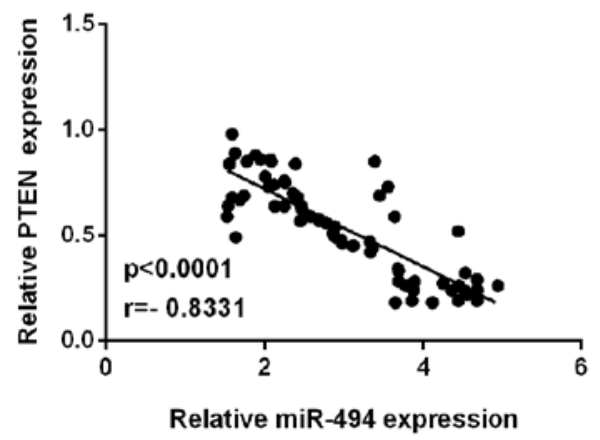

C

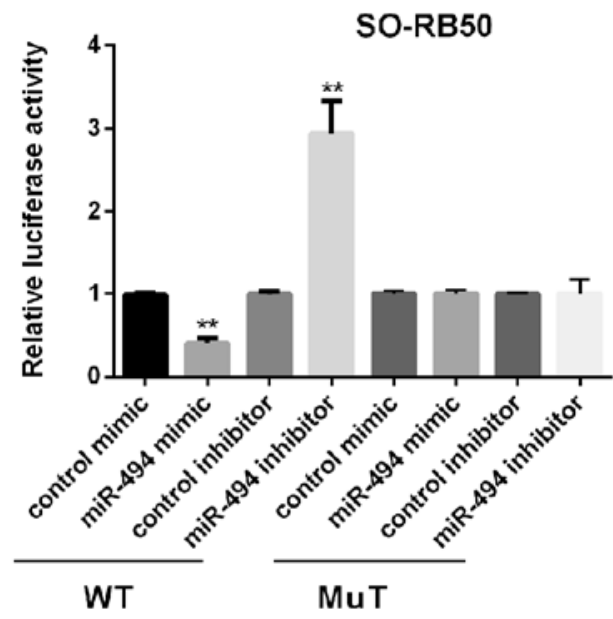

E

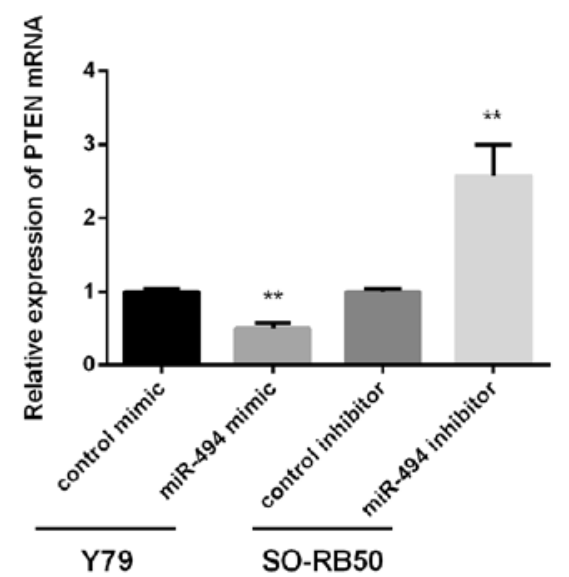

Figure 3. PTEN was the target of miR-494 in RB cells. (A) Schematic representation of the binding sites of miR-494 with PTEN 3'UTR. (B) Relative luciferase activity was tested after overexpression of miR-494 in wild-type (WT) or mutated (MuT) Y79 cells. (C) Relative luciferase activity detected in SO-RB50 cells after silence miR-494 in WT or MuT. (D) Relative protein level of PTEN by western blotting in Y79 cells or SO-RB50 cells. (E) Relative PTEN mRNA expression in Y79 cells or SO-RB50 cells by qRT-PCR. (F) The association of miR-494 expression and PTEN expression was measured by Spearman's Rank correlation. ${ }^{* *} \mathrm{P}<0.01 . \mathrm{RB}$, retinoblastoma; PTEN, phosphatase and tensin homolog.

To investigate whether a change in miR-665 expression affects the PI3K/AKT pathway in RB cells, the expression levels of several important molecules in the pathway, including p-AKT, AKT, p-PI3K and PI3K, were measured in RB cells following upregulation or downregulation of miR-494. As presented in Fig. 5A, miR-494 overexpression remarkably promoted the phosphorylation of PI3K and AKT in Y79 cells. However, silencing miR-494 obviously inhibited the phosphorylation of PI3K and AKT in SO-RB50 cells. Restoration of PTEN reversed effect of miR-494 mimic on PI3K/AKT pathway (Fig. 5B). The results suggested that miR-494 promoted RB cell progression via PTEN/PI3K/AKT signaling pathway. 
A

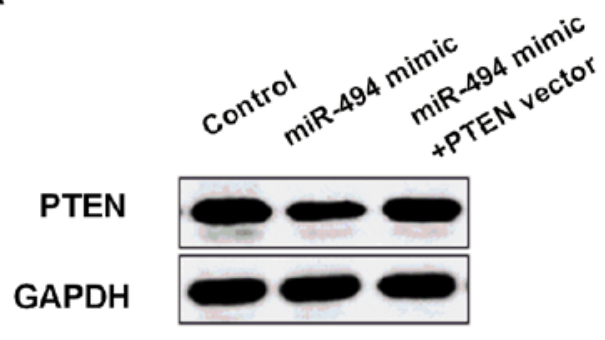

B
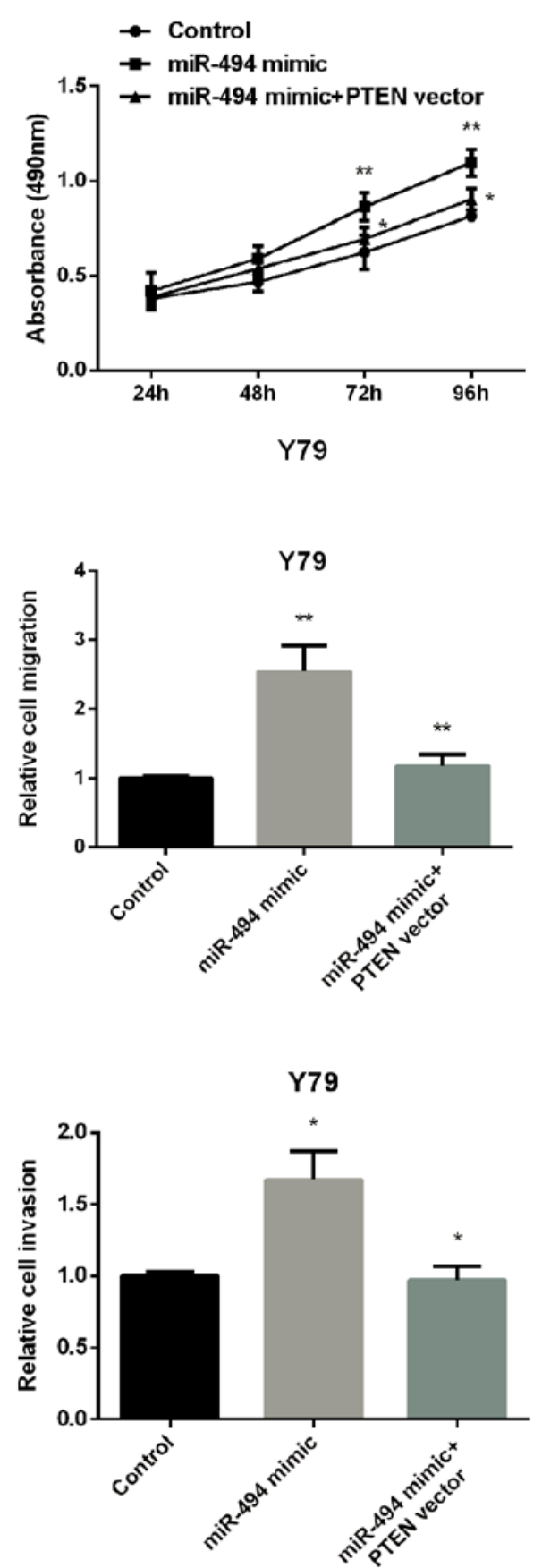

Figure 4. miR-494 regulates RB cell progression via PTEN. (A) Detection of PTEN protein in Y79 cells by western blotting. (B) Cell viability was measured in Y79 cells after increasing miR-494 expression or both miR-494 and PTEN expression. (C) Cell migration was detected in Y79 cells after upregulation of miR-494 or with miR-494 and PTEN. (D) Cell invasion was detected in Y79 cells after upregulation of miR-494 or both miR-494 and PTEN. "P<0.05, ${ }^{* *} \mathrm{P}<0.01$. RB, retinoblastoma; PTEN, phosphatase and tensin homolog.

\section{Discussion}

Herein, we displayed that miR-494 was increased in RB tissue samples and cells, and it was related to the clinicopathological features of RB patients. Moreover, increasing miR-494 facilitated, while inhibiting miR-494, repressed RB cell viability, invasion and migration. Furthermore, PTEN was verified as the target of miR-494 in RB. In addition, it was demonstrated that miR-494 regulated PI3K/AKT signaling pathway by suppressing PTEN in RB cells. Collectively, our findings suggested that overexpression of miR-494 promoted RB cell progression through PTEN/PI3K/AKT axis.

In previous studies, miR-494 has been implicated in the development and progression of various types of tumors. Zhang et al (26) stated that miR-494 played an oncomiR role in lung cancer progression. Zhu et al (25) proved that miR-494 overexpression obviously promoted endometrial cancer cell viability, invasion and migration. Besides, miR-494 mimic significantly enhanced nasopharyngeal carcinoma cell proliferation, invasion and migration (27). In our study, it was shown 
A
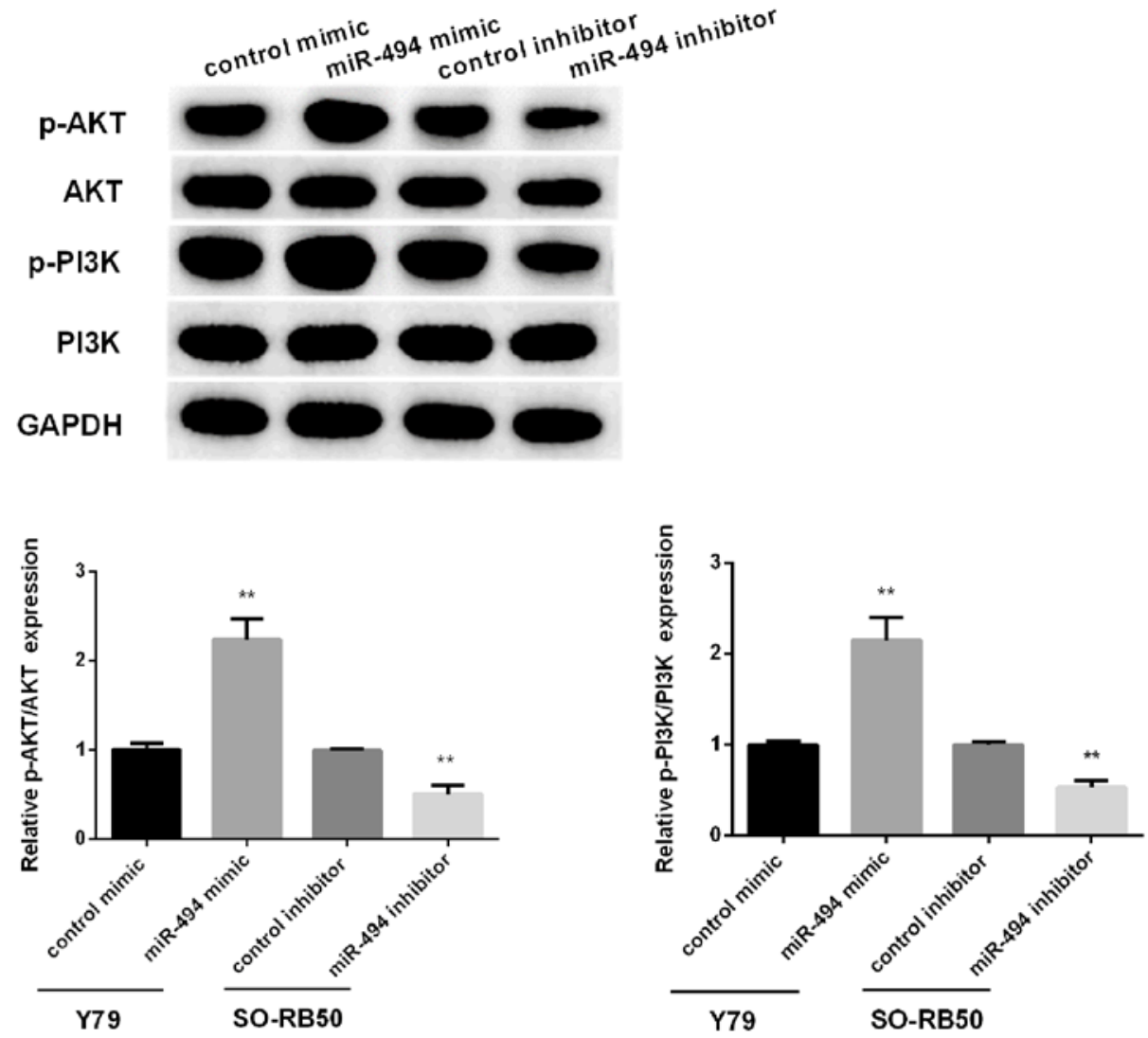

B
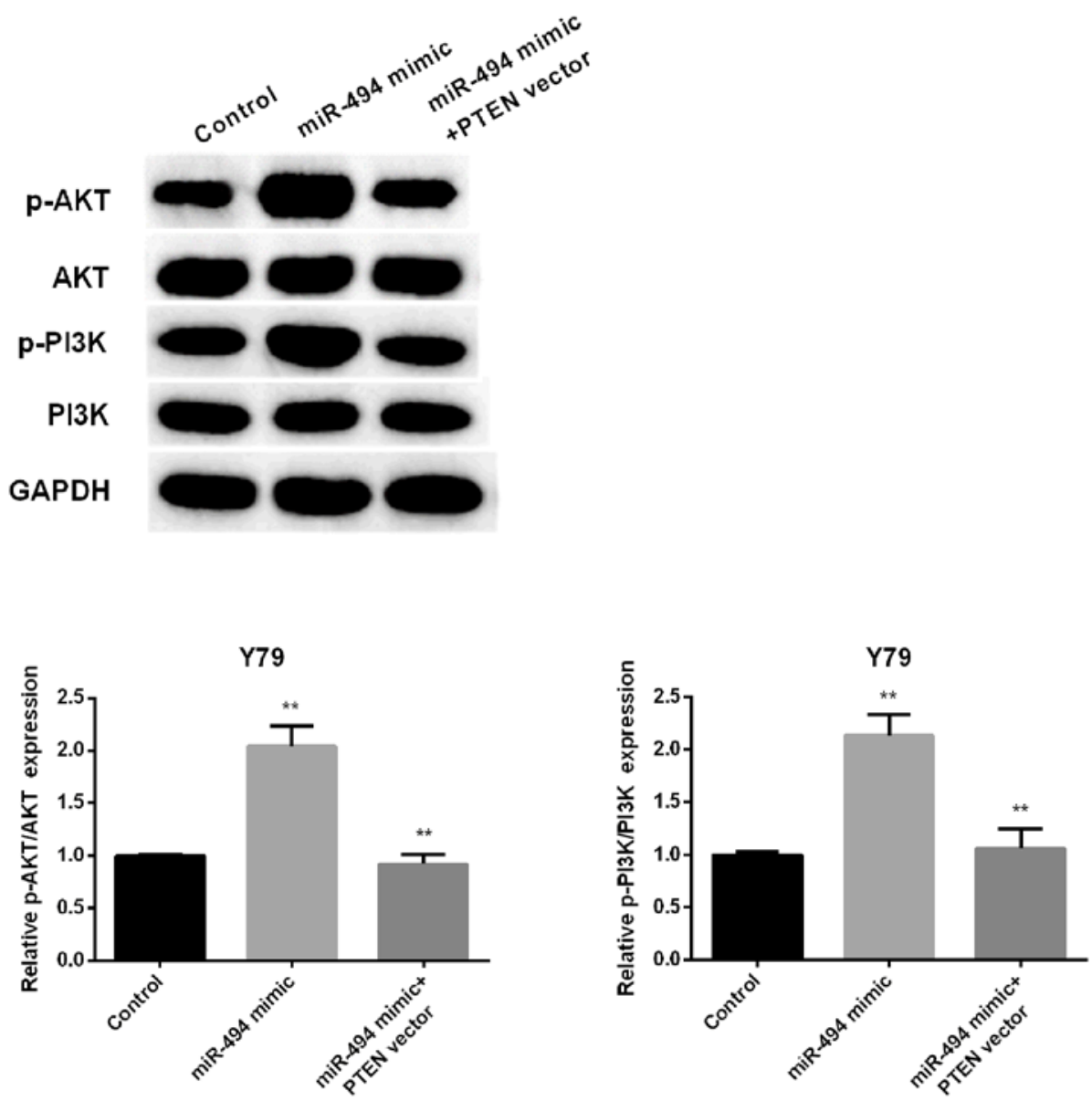

Figure 5. miR-494 regulated PTEN/PI3K/AKT signaling pathway. (A) The expression of p-PI3K/PI3K or p-AKT/AKT in Y79 cells after increasing miR-494 or in SO-RB50 cells after inhibiting miR-494. (B) Quantitative expression of p-PI3K/PI3K or p-AKT/AKT in Y79 cells after overexpression of miR-494 or both miR-494 and PTEN. PTEN, phosphatase and tensin homolog. ${ }^{* *} \mathrm{P}<0.01$. 
that miR-494 functioned as an oncogene in RB progression by enhancing cell viability, invasion and migration. In general, miR-494 played oncomiR roles in multiple cancers via targeting different mRNA genes. For example, miR-494 took part in gastric cancer cell survival by suppressing BAG-1 (28). Cheng et al (29) found that miR-494 repressed cervical cancer cell proliferation, invasion via promoting SOCS6. Zhang et al (30) stated that miR-494 was higher in colorectal cancer and promoted cell progression by targeting APC. In this study we first showed that miR-494 regulated RB development by targeting PTEN, which was the novelty of this research.

PTEN is essential for the maintenance of normal cells and acts as a tumor suppressor in human cancers (31). A large number of studies have reported that PTEN played important roles in tumor development and progression, including lung cancer, ovarian cancer, and prostate cancer $(23,32,33)$. Furthermore, in the RB development, PTEN showed an inhibitory effect and it served as the target of multiple miRNAs. For instance, it was the directly target of miR-93, and miR-198 in promoting human RB progression, and the decreased PTEN expression was also found, which was consists with our findings $(34,35)$. In the present study, it was shown that miR-494 targeted PTEN in regulating RB cell progression, and PTEN overexpression can reverse the effect of miR-494 upregulation in RB cells.

The PI3K/Akt axis is well known to participate in the cell growth and survival of various types of cancers, including RB (36-38). A previous study reported that the ethanol extracts of $\mathrm{R}$. japonica radix (ERJR) could play an inhibitory effect on hepatocellular carcinoma metastasis via PI3K/Akt signaling pathways (39). PTEN is reported to act as an antagonist of PI3K action, it regulates PI3K/Akt signaling negatively (40). For instance, microRNA-155-5p promoted hepatocellular carcinoma progression by suppressing PTEN through the PI3K/Akt pathway (41). Our findings demonstrated that overexpression of miR-494 promoted PI3K/Akt signaling pathways by suppressing PTEN, and PTEN overexpression can reverse the effect of miR-494 upregulation in PI3K/Akt signaling pathways.

Potential limitation of this study is that though miR-494 promoted proliferation of retinoblastoma in our study, this was only verified in RB cell lines, and mouse model might be more convincing. For the experiments using cells transfected with miR-494, using an untransfected control cell line to show that transfection alone did not affect the behavior would be more convincing. Because miR-494 is upregulated and promoted cell viability, invasion and migration in $\mathrm{RB}$, it might be a possible biomarker for predicting progression. In summary, our study indicated that miR-494 acted as an oncogenic microRNA in RB cells by suppressing the tumor suppressor PTEN. miR-494 regulated PI3K/AKT signaling pathway via PTEN.

The present study revealed the important role of miR-494 in promoting the progression of retinoblastoma, although further studies are required to confirm this. Taken together, our research stated that miR-494 overexpression enhanced RB cell progression through PTEN/PI3K/AKT signaling pathway.

\section{Acknowledgements}

Not applicable.

\section{Funding}

No funding was received.

\section{Availability of data and materials}

The datasets used and/or analyzed during the current study are available from the corresponding author on reasonable request.

\section{Authors' contributions}

FX conceived the study and drafted the manuscript. FX, GL and LW were responsible for cell culture, qRT-PCR and western blot analysis. XW, XJ and WB performed MTT assay and luciferase assay. All authors read and approved the final manuscript.

\section{Ethics approval and consent to participate}

This study was approved by the Ethics Committee of Jinan Zhangqiu District Hospital of TCM (Jinan, China). Patients who participated in this research had complete clinical data. Parents of the child patients signed the informed consent form.

\section{Patient consent for publication}

Not applicable.

\section{Competing interests}

The authors declare that they have no competing interests.

\section{References}

1. Dimaras H, Dimba EA and Gallie BL: Challenging the global retinoblastoma survival disparity through a collaborative research effort. Br J Ophthalmol 94: 1415-1416, 2010.

2. Kivelä T: The epidemiological challenge of the most frequent eye cancer: Retinoblastoma, an issue of birth and death. Br J Ophthalmol 93: 1129-1131, 2009.

3. Narang S, Mashayekhi A, Rudich D and Shields CL: Predictors of long-term visual outcome after chemoreduction for management of intraocular retinoblastoma. Clin Exp Ophthalmol 40: 736-742, 2012.

4. Shinohara ET, DeWees T and Perkins SM: Subsequent malignancies and their effect on survival in patients with retinoblastoma. Pediatr Blood Cancer 61: 116-119, 2014.

5. Stupp R, Mason WP, van den Bent MJ, Weller M, Fisher B, Taphoorn MJ, Belanger K, Brandes AA, Marosi C, Bogdahn U, et al; European Organisation for Research and Treatment of Cancer Brain Tumor and Radiotherapy Groups; National Cancer Institute of Canada Clinical Trials Group: Radiotherapy plus concomitant and adjuvant temozolomide for glioblastoma. N Engl J Med 352: 987-996, 2005.

6. Holland EC: Gliomagenesis: Genetic alterations and mouse models. Nat Rev Genet 2: 120-129, 2001.

7. Liu S, Hu C, Wang Y, Shi G, Li Y and Wu H: miR-124 inhibits proliferation and invasion of human retinoblastoma cells by targeting STAT3. Oncol Rep 36: 2398-2404, 2016.

8. Wang L, Lyu X, Ma Y, Wu F and Wang L: MicroRNA 504 targets AEG 1 and inhibits cell proliferation and invasion in retinoblastoma. Mol Med Rep 19: 2935-2942, 2019.

9. Guo L, Bai Y, Ji S and Ma H: MicroRNA 98 suppresses cell growth and invasion of retinoblastoma via targeting the IGF1R/k Ras/Raf/ MEK/ERK signaling pathway. Int J Oncol 54: 807-820, 2019.

10. Golabchi K, Soleimani-Jelodar R, Aghadoost N, Momeni F, Moridikia A, Nahand JS, Masoudifar A, Razmjoo H and Mirzaei H: MicroRNAs in retinoblastoma: Potential diagnostic and therapeutic biomarkers. J Cell Physiol 233: 3016-3023, 2018. 
11. Yu F, Pang G and Zhao G: ANRIL acts as onco-lncRNA by regulation of microRNA-24/c-Myc, MEK/ERK and Wnt/ $\beta$-catenin pathway in retinoblastoma. Int J Biol Macromol 128: 583-592, 2019.

12. Sun Z, Zhang A and Zhang L: Inhibition of microRNA 492 attenuates cell proliferation and invasion in retinoblastoma via directly targeting LATS2. Mol Med Rep 19: 1965-1971, 2019.

13. Wang J, Wang X, Li Z, Liu H and Teng Y: MicroRNA-183 suppresses retinoblastoma cell growth, invasion and migration by targeting LRP6. FEBS J 281: 1355-1365, 2014.

14. Dalgard CL, Gonzalez M, deNiro JE and O'Brien JM: Differential microRNA-34a expression and tumor suppressor function in retinoblastoma cells. Invest Ophthalmol Vis Sci 50: 4542-4551, 2009.

15. Zhao JJ, Yang J, Lin J, Yao N, Zhu Y, Zheng J, Xu J, Cheng JQ Lin JY and Ma X: Identification of miRNAs associated with tumorigenesis of retinoblastoma by miRNA microarray analysis Childs Nerv Syst 25: 13-20, 2009.

16. Li J, Yen C, Liaw D, Podsypanina K, Bose S, Wang SI, Puc J, Miliaresis C, Rodgers L, McCombie R, et al: PTEN, a putative protein tyrosine phosphatase gene mutated in human brain, breast, and prostate cancer. Science 275: 1943-1947, 1997.

17. Tay Y, Kats L, Salmena L, Weiss D, Tan SM, Ala U, Karreth F, Poliseno L, Provero P, Di Cunto F, et al: Coding-independent regulation of the tumor suppressor PTEN by competing endogenous mRNAs. Cell 147: 344-357, 2011.

18. Xu W, Yang Z, Zhou SF and Lu N: Posttranslational regulation of phosphatase and tensin homolog (PTEN) and its functional impact on cancer behaviors. Drug Des Devel Ther 8: 1745-1751, 2014.

19. Wu W, Yang J, Feng X, Wang H, Ye S, Yang P, Tan W, Wei G and Zhou Y: MicroRNA-32 (miR-32) regulates phosphatase and tensin homologue (PTEN) expression and promotes growth, migration, and invasion in colorectal carcinoma cells. Mol Cancer 12: 30, 2013.

20. Wang ZX, Lu BB, Wang H, Cheng ZX and Yin YM: MicroRNA-21 modulates chemosensitivity of breast cancer cells to doxorubicin by targeting PTEN. Arch Med Res 42: 281-290, 2011.

21. Ma F, Zhang J, Zhong L, Wang L, Liu Y, Wang Y, Peng L and Guo B: Upregulated microRNA-301a in breast cancer promotes tumor metastasis by targeting PTEN and activating Wnt// $/$-catenin signaling. Gene 535: 191-197, 2014.

22. Rong D, Lu C, Zhang B, Fu K, Zhao S, Tang W and Cao H: CircPSMC3 suppresses the proliferation and metastasis of gastric cancer by acting as a competitive endogenous RNA through sponging miR-296-5p. Mol Cancer 18: 25, 2019.

23. Akgun S, Kucuksayan H, Ozes ON, Can O, Alikanoglu AS, Yildiz $M$ and Akca $H$ : NF- $\mathrm{B}$-induced upregulation of miR-548as-3p increases invasion of NSCLC by targeting PTEN Anticancer Agents Med Chem 19: 1058-1068, 2019.

24. Zhao YS, Yang WC, Xin HW, Han JX and Ma SG: MiR-182-5p knockdown targeting PTEN inhibits cell proliferation and invasion of breast cancer cells. Yonsei Med J 60: 148-157, 2019.

25. Zhu L, Wang X, Wang T, Zhu W and Zhou X: miR-494-3p promotes the progression of endometrial cancer by regulating the PTEN/PI3K/AKT pathway. Mol Med Rep 19: 581-588, 2019.

26. Zhang Q, Li Y, Zhao M, Lin H, Wang W, Li D, Cui W, Zhou C, Zhong J and Huang C: MiR-494 acts as a tumor promoter by targeting CASP2 in non-small cell lung cancer. Sci Rep 9: 3008, 2019.

27. He H, Liao X, Yang Q, Liu Y, Peng Y, Zhong H, Yang J, Zhang H, Yu Z, Zuo Y, et al: MicroRNA-494-3p promotes cell growth, migration, and invasion of nasopharyngeal carcinoma by targeting Sox7. Technol Cancer Res Treat: Jan 1, 2018 (Epub ahead of print). doi: $10.1177 / 1533033818809993$
28. Shen Z, Li Y, Zhao C, Wang F, Zhou R and Chen G: miR-494 BAG 1 axis is involved in cinobufacini induced cell proliferation and apoptosis in gastric cancer. Mol Med Rep 17: 7435-7441, 2018.

29. Cheng L, Kong B, Zhao Y and Jiang J: miR-494 inhibits cervical cancer cell proliferation through upregulation of SOCS6 expression. Oncol Lett 15: 3075-3080, 2018.

30. Zhang Y, Guo L, Li Y, Feng GH, Teng F, Li W and Zhou Q: MicroRNA-494 promotes cancer progression and targets adenomatous polyposis coli in colorectal cancer. Mol Cancer 17: 1, 2018.

31. Lee YR, Chen M and Pandolfi PP: The functions and regulation of the PTEN tumour suppressor: New modes and prospects. Nat Rev Mol Cell Biol 19: 547-562, 2018.

32. Schroeder C, Grell J, Hube-Magg C, Kluth M, Lang D, Simon R, Höflmayer D, Minner S, Burandt E, Clauditz TS, et al: Aberrant expression of the microtubule-associated protein tau is an independent prognostic feature in prostate cancer. BMC Cancer 19: 193, 2019

33. Kim TH, Park JH and Woo JS: Resveratrol induces cell death through ROS dependent downregulation of Notch1/PTEN/Akt signaling in ovarian cancer cells. Mol Med Rep 19: 3353-3360, 2019.

34. Cao Y, Xia F, Wang P and Gao M: MicroRNA 93-5p promotes the progression of human retinoblastoma by regulating the PTEN/PI3K/AKT signaling pathway. Mol Med Rep 18: 5807-5814, 2018.

35. Wei D, Miao Y, Yu L, Wang D and Wang Y: Downregulation of microRNA-198 suppresses cell proliferation and invasion in retinoblastoma by directly targeting PTEN. Mol Med Rep 18: 595-602, 2018

36. Lin A, Piao HL, Zhuang L, Sarbassov D, Ma L and Gan B: FoxO transcription factors promote AKT Ser473 phosphorylation and renal tumor growth in response to pharmacologic inhibition of the PI3K-AKT pathway. Cancer Res 74: 1682-1693, 2014.

37. Spangle JM, Roberts TM and Zhao JJ: The emerging role of PI3K/AKT-mediated epigenetic regulation in cancer. Biochim Biophys Acta Rev Cancer 1868: 123-131, 2017.

38. Xie C, Lu H, Nomura A, Hanse EA, Forster CL, Parker JB, Linden MA, Karasch C and Hallstrom TC: Co-deleting Pten with $\mathrm{Rb}$ in retinal progenitor cells in mice results in fully penetrant bilateral retinoblastomas. Mol Cancer 14: 93, 2015.

39. Kim BR, Ha J, Lee S, Park J and Cho S: Anti-cancer effects of ethanol extract of Reynoutria japonica Houtt. radix in human hepatocellular carcinoma cells via inhibition of MAPK and PI3K/Akt signaling pathways. J Ethnopharmacol 245: 112179, 2019.

40. Haddadi N, Lin Y, Travis G, Simpson AM, Nassif NT and McGowan EM: PTEN/PTENP1: 'Regulating the regulator of RTK-dependent PI3K/Akt signalling', new targets for cancer therapy. Mol Cancer 17: 37, 2018.

41. Fu X, Wen H, Jing L, Yang Y, Wang W, Liang X, Nan K, Yao Y and Tian T: MicroRNA-155-5p promotes hepatocellular carcinoma progression by suppressing PTEN through the PI3K/Akt pathway. Cancer Sci 108: 620-631, 2017.

This work is licensed under a Creative Commons Attribution-NonCommercial-NoDerivatives 4.0 International (CC BY-NC-ND 4.0) License. 